\title{
Rursus
}

Russus

Poiétique, réception et réécriture des textes antiques

$3 \mid 2008$

Varia

\section{Parcours et vertus de Saint Martin dans la Vita et les Epistulae de Sulpice Sévère}

Anne-Marie Taisne

\section{OpenEdition}

Journals

Édition électronique

URL : http://journals.openedition.org/rursus/220

DOI : $10.4000 /$ rursus.220

ISSN : 1951-669X

Éditeur

Université Nice-Sophia Antipolis

Référence électronique

Anne-Marie Taisne, «Parcours et vertus de Saint Martin dans la Vita et les Epistulae de Sulpice Sévère », Rursus [En ligne], $3 \mid$ 2008, mis en ligne le 15 février 2008, consulté le 20 avril 2019. URL : http:// journals.openedition.org/rursus/220 ; DOI : 10.4000/rursus.220

Ce document a été généré automatiquement le 20 avril 2019.

Rursus 


\title{
Parcours et vertus de Saint Martin dans la Vita et les Epistulae de Sulpice Sévère
}

\author{
Anne-Marie Taisne
}

1 Quand au printemps 397 paraît la Vita Martini de Sulpice Sévère, précédant de peu la mort du Saint le 11 novembre de la même année, l'Empire romain et l'Église chrétienne viennent de connaître une période paradoxale où leur histoire apparaît encore intrinsèquement imbriquée, même si celle qui représente la Cité de Dieu ici-bas connaît un épanouissement et une extension extraordinaires tandis que la Cité terrestre, l'Empire romain, entre le règne de Constantin et celui de Théodose qui ont réunifié l'Empire, connait nombre de bouleverse-ments dus aux guerres civiles et aux pressions des Barbares à l'Ouest et à l'Est.

2 Constantin, en effet, a réuni l'Empire après avoir successivement battu Maxence au pont Milvius (312), puis Licinius (324). Théodose, après avoir vaincu Maxime (379), règnera jusqu'en 395. Entre temps, les Alamans avaient franchi le Rhin et avaient été battus par Julien en 357. La guerre en Asie avait sévi de 360 à 363. En 378, Valens mourait à Andrinople sous les assauts des Wisigoths. En somme, la Pax Romana était menacée à l'intérieur comme à l'extérieur : on comprend dès lors l'importance prise par l'armée à cette époque.

3 Cette période a été cependant souvent qualifiée d'âge d'or pour le Christianisme ${ }^{1}$. En effet, le début du siècle a vu la fin des persécutions en 313 lors de ce qu'on a appelé «l'Edit de Milan» qui a permis la liberté des cultes dans l'Empire et a vu l'avènement de princes chrétiens dont Constantin est le premier représentant. Toutefois la paix de l'Eglise est compromise à la fois par l'arianisme et le règne de Julien dit «l'apostat » (361-363). Malgré tout, l'extension du Christianisme est considérable, «l'Eglise vit au grand jour» (Testard). Les lieux de culte se multiplient tandis que les fastes de la liturgie se développent. La ferveur populaire s'accroît et l'aristocratie est amplement convertie, parfois même attirée par le monachisme, tandis que l'approfondissement doctrinal de l'Eglise suscite nombre de conciles et de synodes locaux. 
4 C'est dans un tel climat que, selon l'expression de J. Fontaine (S.E.L. 12 Fév. 05) on atteint « les cimes de la production littéraire de l'Antiquité tardive». Chez les auteurs chrétiens, qu'il nous suffise de citer quelques grands noms parmi les Pères de l'Eglise : Saint Hilaire de Poitiers (湢 315-367), Saint Ambroise de Milan (339 ?-397), Saint Jérôme (347-419) qui à partir de 385 se fixera en Orient et Saint Augustin qui deviendra évêque d'Hippone (354-430). Dans l'Occident latin, ce IV s. voit le christianisme s'implanter solidement dans toutes les provinces de l'Empire. De plus, les mesures anti-païennes vont s'accroître : en 380, l'Edit de Thessalonique impose la foi romaine et porte aux autres religions une condamnation de principe ; de 386 à 391 plusieurs édits de Théodose vont persécuter le paganisme : les célébrations seront interdites en public, les sanctuaires et les statues des païens seront détruits; en 393 même les Jeux Olympiques seront abolis. Toutefois la conversion des campagnes ne se fait pas sans difficultés. Une partie de l'aristocratie aussi reste attachée à la vieille tradition religieuse, surtout les Sénateurs.

5 L'affaire de l'Autel de la Victoire à Rome symbolise particulièrement la confrontation entre chrétiens et païens. De 384 à 394, l'autel de la Victoire qui, depuis Auguste, était placé dans la Curie à Rome amène une confrontation entre Symmaque, préfet de Rome, et Saint Ambroise : l'autel sera enlevé, puis replacé, avant d'être enlevé définitivement. La cause chrétienne prévalut, comme le confirme le Contre Symmaque du poète Prudence.

6 La foi chrétienne se manifeste chez des écrivains laïques : en poésie, de manière assez discrète chez Ausone, mais de façon très engagée chez Prudence. Et c'est la ferveur d'un chrétien laïque qui a fait connaître les événements majeurs de la vie d'un Saint de ce siècle qui, pour sa part, n'a laissé aucun écrit, mais est devenu l'objet d'un culte déjà de son vivant, et encore plus à partir de sa mort. Il s'agit de Saint Martin, autour de la vie duquel s'articule la majeure partie de l'œuvre de Sulpice Sévère, notamment la Vita et les Epistulae.

7 Qui était Sulpice Sévère? Nous connaissons sa biographie par quelques anecdotes à travers son œuvre, mais surtout grâce à la correspondance échangée avec Paulin, son ancien condisciple à l'Université ; Sulpice était né en Aquitaine dans les années 360, issu d'une riche famille de l'aristocratie gallo-romaine. Sa position sociale fut encore accrue par son mariage avec la fille d'un consulaire et d'une noble dame du nom de Bassula. Il fit ses études à Bordeaux dont l'Université connaissait alors un grand rayonnement. C'est là que Paulin de Nole avait fait ses études et connu une belle carrière avant de renoncer aux biens matériels. Dans cette Aquitaine où la culture romaine était florissante, Sulpice acquit la célébrité comme avocat -Paulin admirait son talent oratoire dès l'Université- et ceci explique parfois l'aspect apologétique de son œuvre. Si l'on ignore la date de son baptême, on sait qu'il se convertit à la vie ascétique à partir de 393 sans doute du fait de la mort précoce de sa femme et sous l'influence de sa belle-mère Bassula, elle-même fervente de l'ascétisme. "Saisi d'une impulsion soudaine » (L. Pietri, p.2) il commence à se dépouiller pour ne plus songer qu'à " l'accroissement de son patrimoine céleste » et se retire dans son domaine de Primuliacum. L'influence conjuguée de Martin et de son ami Paulin favorisa largement cette « conversion ».

8 La retraite de Sulpice à Primuliacum, comme l'a suggéré J.Fontaine, est une sorte de variante chrétienne du secessus ad uillam des Romains lettrés. Cette vie ascétique est d'ailleurs «tempérée » car, si Sulpice et ses amis logent bien dans des cellules, couchent sur des paillasses et se vêtent de palliums en poil de chameau, cette vie bénéficie des avantages d'une riche demeure aristocratique. La solitude est rompue par la visite des propriétaires des alentours et des voyageurs venus de Tours et d'Orient (cf les Dialogues). 
Si les entretiens restent pieux et centrés sur la personne de Martin, la culture classique n'en est point bannie chez des lettrés qui se veulent à la fois Christiani et Ciceroniani (cf. Jérôme, Ep. 22).

9 C'est dans cette retraite que Sulpice rédigea notamment la Vita Martini qui parut en 397, ainsi que les 3 Epitres adressées à des admirateurs de Martin, le prêtre Eusèbe, le diacre Aurèle, sa belle-mère Bassula en 397 et 398. Quelques années plus tard (403-404) furent rédigés les Dialogues entre l'auteur, Postumianus et Gallus, sans compter les Chroniques sur l'histoire de l'univers dont la fin du L. II (400-404) est consacrée à l'affaire priscillianiste et au rôle qu'y joua Martin. Le but de Sulpice Sévère est avant tout dans la majorité de son œuvre de faire connaître la vie du Saint et ses mérites.

10 Avant la parution de la Vita Martini, la Vie de Saint Antoine, un ermite de Haute-Égypte, avait été rédigée par Athanase, l'évêque d'Alexandrie, puis traduite du grec en latin par Evagrius d'Antioche avant 388. Saint Augustin rapporte précisément comment la lecture de l'ouvrage avait suscité des vocations de vie ascétique jusque dans l'empire d'Occident ( Conf. VIII) : il constate, comme le rappelle P. Brown (1971: 185), la multitude des monastères qui s'organisent autour de Milan et le grand nombre de «ceux que les carrières normales et les liens traditionnels de classe et d'éducation ne parvenaient plus à retenir ». En évoquant les exploits d'Antoine et ceux de Martin, J. Fontaine parle d'un " concours de sainteté ». En effet, tout comme Athanase l'avait fait pour Saint Antoine en Orient, Sulpice veut donner à l'Occident l'image d'un Saint hors du commun ${ }^{2}$. Mais Martin en Occident égalera et même surpassera les ascètes orientaux comme le confirmeront les Dialogues. Son ami Paulin écrit à Sulpice à propos de la Vita qu'il vient de lire : «Tu es donc béni du Seigneur pour avoir relaté, avec autant de dignité dans le style que de justesse dans les sentiments, l'histoire d'un si grand évêque et d'un confesseur aussi éclatant. Mais bienheureux, lui aussi, en récompense de ses mérites, pour avoir mérité un historien digne de sa foi et de sa vie, et de se trouver ainsi doublement consacré à la gloire divine par ses mérites, à la mémoire des hommes par tes écrits » $(E p .11,11)$.

Cet éloge de Paulin correspond aux desseins mêmes de Sulpice qui, d'après son ami, a appliqué les règles de l'aptum, où la forme est adaptée au fond. L'éloge christianise un thème classique illustré notamment par Horace qui soulignait dans l'Ode à Auguste (4.14.3 sq.) l'importance d'un bon écrivain pour relater la vie d'un grand homme, uirtutes aeternare. Sulpice lui-même nous confie dans ses œuvres ses propres ambitions; dans la Vita, c'est surtout dans les préliminaires et dans la conclusion.

12 La lettre de dédicace à Didier, sans doute un aristocrate converti à l'ascétisme et connu aussi de Paulin, se conforme aux lois du genre épistolaire avec le salut au début, l'adieu à la fin, et comporte à chaque fois une déclaration d'amitié. La profession d'humilité de l'auteur reste conforme aux topoi de la rhétorique prônés par Cicéron et Quintilien: l'ouvrage est qualifié de libellus, diminutif qui accentue cette modestie affichée; puis Sulpice emprunte à Horace la métaphore de la séquestration qui compare l'œuvre à un esclave qui s'échappe pour arriver jusqu'aux lecteurs. Ensuite l'auteur affirme la prépondérance des res sur les uerba en opposant l'éloquence à la foi selon un procédé que l'on retrouve notamment chez deux illustres de ses coreligionnaires contemporains, Saint Jérôme et Saint Augustin; il met en parallèle les orateurs et les pêcheurs, évoquant ainsi l'humble origine de certains apôtres qui ont transmis l'Evangile. Toutefois il considère comme un devoir de piété d'exposer les «vertus d'un si grand homme», reprend sa profession d'humilité pour évoquer ses études peu approfondies et dont il s'est 
déshabitué, enfin il tend même à s'effacer, au propre et au figuré, devant son sujet dont seul le héros est important.

Dans la Préface, Sulpice reprend les thèmes traditionnels exposés par nombre d'écrivains profanes tout en soulignant l'originalité d'un dessein chrétien. Même si les écrivains profanes avaient de nobles desseins en proposant une historia magistra uitae (Cicéron), notamment dans les exemples tirés de la vie des grands hommes, et désiraient ainsi acquérir l'immortalité (on pense notamment aux Préfaces d'un Salluste ou d'un TiteLive), pour Sulpice ce type de gloire est éphémère et s'oppose à « la vie bienheureuse et éternelle » à laquelle aspirent les chrétiens. Il évoque encore deux branches maîtresses de l'éducation romaine: la poésie épique avec «les combats d'Hector» qui évoquent Homère, et la philosophie avec « les entretiens de Socrate » qui désignent Platon. Il parle à la façon des Apologistes de "fables " et de "tombeaux " pour désigner l'erreur des païens, et il oppose à un fol héroïsme et à une vaine philosophie l'idéal du chrétien et du saint qui veulent vivre pieusement, saintement et religieusement. Son œuvre sera utile car elle suscitera chez le lecteur une émulation pour accéder à la vraie sagesse, à la milice céleste et à la vertu divine. On retrouve ici la christianisation de l'idéal profane des exempla. D'autre part, rejoignant l'idéal d'un Ammien Marcellin, Sulpice a opéré un choix parmi les faits « les plus saillants » pour ne pas lasser le lecteur, et il insiste sur son désir de s'attacher à la vérité, comme un témoin ferait une déclaration à la barre. Au fil de la narration, ces desseins de l'auteur (Vita 19.5: souci de la vérité et d'éviter la lassitude; Vita 22.6 : valeur d'exemple d'une anecdote ; Vita 24.8 : véracité des faits).

Dans la fin et dans la conclusion de la Vita, il affiche à nouveau sa modestie, en s'accusant de maladresse et d'infériorité par rapport au sujet, en reprenant le thème classique de l'impossibilité du discours à traduire certaines vérités intérieures (26.1). Il a des mots très durs en conclusion pour les détracteurs et les persécuteurs de Martin, en employant à leur sujet des images péjoratives très fortes telles que « cruels serpents » et «chiens aux abois ». Il oppose les saintes personnes qui liront son ouvrage avec plaisir aux incrédules qui auront tort. Le désir d'authenticité et l'amour du Christ l'ont inspiré. Une récompense divine sera accordée aux croyants.

Dans les épîtres qui suivent la publication de la Vita, on retrouve quelques-unes des constatations faites sur son dessein littéraire : ainsi dans la Lettre à Eusèbe, après avoir fait une allusion au succès de son libellus (1.1), il rappelle le choix de ne mentionner que les faits les plus importants et l'impossibilité d'embrasser tous les actes du Saint (1.8); dans la Lettre à Bassula, il revient sur « la tâche écrasante de faire connaître par la plume tout ce que l'on doit savoir de Martin ».

Comment Sulpice réalise-t-il son dessein? Nous le vérifierons en examinant d'abord les principales étapes de son parcours dans la Vita et les Epîtres.

Les sept chapitres qui sont consacrés à la première partie de la vie de Martin (2 à 8) nous montrent les différentes étapes de sa vie de chrétien, du catéchuménat à l'épiscopat en passant par le baptême, sa nomination comme exorciste, ses nombreux voyages en Gaule et en Italie, avec quelques haltes dans des ermitages, animé qu'il est d'une foi indomptable qui entraîne des conversions et qui résiste à l'hérésie arienne. Les voyages qui ponctuent sa jeunesse sont liés à la vie de son père, tribun tout d'abord, puisque sa pueritia se déroule de Sabaria en Pannonie à Pavie en Italie; puis, à partir de 15 ans, son adulescentia et le début de sa maturité le conduiront à suivre l'armée, où il servira dans la cavalerie de la garde avec une étape signalée à Amiens et une autre au sud de Trèves. Sulpice souligne les aspirations de Martin à la vie chrétienne dès son âge tendre, puisqu'à 
10 ans il demande le catéchuménat, montrant déjà des qualités qui seront illustrées à l'adolescence dans l'épisode du manteau partagé (ch. 3) ; il a 18 ans quand « il s'empressa de se faire baptiser" (3.5). Sulpice passe sous silence les longues années de service militaire; en revanche, il campe en une scène dramatique le moment où, non loin de Trèves, à la veille d'une bataille contre les Barbares, Martin obtient du César Julien son congé en proposant même à son chef une sorte de deuotio à la manière chrétienne ${ }^{3}$. évêque en $350^{4}$, qui le nomme exorciste. Puis, à la suite d'un songe, Martin repart dans sa patrie pour convertir ses parents. Il se sépare de l'évêque avec tristesse, prévoyant les épreuves qui l'attendent. De fait, pendant la traversée des Alpes, il est pris par des brigands ; à celui qui lui demande son identité, il répond qu'il est chrétien, puis se montre tellement ferme et persuasif qu'il convertit le brigand qui le détenait et qui rendit témoignage de ce fait (ch. 5). Après Milan, Martin rencontre le diable qui prend figure humaine: il s'ensuit une déclaration de guerre qui, en effet, inaugure une série de combats futurs de l'exorciste Martin aux prises avec le démon qu'il qualifie ici d' « ennemi » (6.1-2). Arrivé chez lui, Martin convertit sa mère, mais non son père (6.3).

Un autre combat va être mené âprement par Martin en Illyrie : celui contre les Ariens, les disciples de l'Alexandrin Arius qui refusait au Christ la substance divine du Père et dont la doctrine avait été condamnée en 325 à Nicée (Hilaire à Poitiers, Ambroise à Milan et Augustin à Hippone combattirent avec force cette hérésie). Persécuté par ces hérétiques, Martin revient en Italie où il vit dans un ermitage à Milan jusqu'à ce que l'arien Auxence le chasse ; s'étant alors retiré dans l'île de Gallinara où il se guérit par ses prières, puis il part pour Rome pour retrouver Hilaire $(6,4-7)$.

Ayant retrouvé Hilaire à Poitiers, il fonde un ermitage à Ligugé et s'illustre alors par un premier miracle qu'il obtient par ses prières et sa foi en Dieu: il ressuscite un catéchumène (ch. 7), puis un jeune esclave (ch. 8). Le chapitre 9 couronne la première partie de la Vita : on y voit Martin emmené par une foule enthousiaste de Poitiers à Tours pour y être nommé évêque malgré « l'opposition impie de quelques évêques ».

21 Toutefois Martin, tout en remplissant ses fonctions d'évêque, se préoccupe de fonder l'ermitage de Marmoutier un peu à l'écart de la ville: les moines, parmi lesquels se trouvent de nombreux nobles et de futurs évêques, sont soumis à une règle de vie faite d'humilité (ch.10). Cinq chapitres (11-15) illustrent la lutte acharnée de Martin à Tours et dans sa région contre la superstition et le paganisme encore vivaces dans les campagnes : chaque chapitre illustre certes l'action d'un saint thaumaturge, mais qui sait aussi convaincre par sa parole; de ce fait, si la destruction des sanctuaires et des idoles des païens est constante, églises et ermitages les remplacent aussitôt. On voit Martin évêque voyager à nouveau notamment à Trèves (ch. 16) et à Paris (ch. 18) où se manifeste sa gratia curationum. Sa lutte contre le démon reste particulièrement intense.

Très émouvant est le chapitre 25 qui, peu avant la conclusion, relate la rencontre entre Sulpice et Martin où le saint prescrit à ses visiteurs le renoncement aux biens matériels en donnant Paulin en exemple. Dans les 2 derniers chapitres, Sulpice insiste encore sur les qualités extraordinaires pratiquées constamment par Martin, ce qui rend d'autant plus odieux ses détracteurs et les incrédules, alors que Dieu accordera sa récompense à ceux qui auront cru (ch. 26-27). Les 3 lettres rédigées après la mort du saint apportent quelques compléments importants à la Vita: la première concernant à nouveau ses pouvoirs de thaumaturge et les deux autres nous renseignant surtout amplement sur sa mort. 
23 La lettre à Eusèbe revient d'abord sur le succès qu'a connu la Vita dès sa publication. Sulpice reprend un thème déjà présent dans la Vita à savoir l'impossibilité d'inclure tous les faits et geste du saint dans un volume. Mais la première partie de la lettre laisse exploser l'indignation de l'auteur au sujet des propos ironiques tenus par un individu sur les brûlures dont Martin n'aurait pas su se protéger : à cette calomnie Sulpice répond, dans un premier temps, par des propos virulents contre ce qu'il considère comme un blasphème. Il rappelle à ce sujet les Juifs insultant le Christ en croix, puis il met en parallèle Martin et les apôtres qui ont couru des dangers comme Paul et Pierre, ce qui lui permet au passage d'accuser le calomniateur d'ignorer l'Ecriture Sainte. A partir de ces exemples fameux, l'auteur affirme que c'est le propre des saints que d'affronter les pires épreuves et d'en sortir vainqueur. Le deuxième volet de la lettre rétablit la vérité en narrant les circonstances lors desquelles Martin fut en partie atteint par le feu dans la sacristie d'une église car il avait tardé à combattre le danger par ses armes habituelles, la foi et la prière. Sulpice conclut en constatant que la sainteté de Martin fut prouvée une fois de plus à cette occasion.

Les deux lettres suivantes, celle au diacre Aurèle et celle à Bassula apportent un précieux complément à la Vita en ce sens qu'elles concernent les derniers moments du saint et nous permettent de mieux cerner quelques traits au sujet de Sulpice lui-même.

La lettre à Aurèle nous montre Sulpice méditant dans sa cellule sur l'espérance des choses à venir ; ses autres pensées sont pessimistes et consistent en dégoût du présent, crainte du jugement, peur du châtiment, conscience de ses fautes. Mais dans le sommeil qui suit une telle veille, voici qu'une apparition se présente : c'est Martin tout resplendissant, mais reconnaissable, tenant à la main la Vita, tandis qu'il bénit Sulpice en répétant le nom de la croix; puis il disparaît dans les cieux entr'ouverts suivi de près par son disciple Clair, décédé depuis peu. S'efforçant de les suivre, Sulpice se réveille en sursaut : c'est pour apprendre aussitôt le décès de Martin. Comme dans les chants de deuil, le ton de Sulpice se fait pathétique pour évoquer la profonde douleur qui le saisit alors; il appelle au secours son ami fidèle pour partager sa peine; suit un fervent éloge de Martin qui a rejoint désormais le " glorieux troupeau des justes »; sa vie dans l'au-delà est peinte aux couleurs de l'Apocalypse. En des termes enthousiastes, qui ne sont pas sans évoquer certains Hymnes du Péristéphanon Liber de son contemporain Prudence, Sulpice démontre que, sans verser son sang, Martin a atteint néanmoins «la plénitude du martyr »; il évoque les persécutions de Néron et de Dèce en suggérant différents supplices que Tertullien avait déjà énumérés en référence à des supplices fameux de l'Ancien Testament- les jeunes Hébreux dans la fournaise et Isaïe torturé-, en revenant à saint Paul torturé; aux yeux de Sulpice qui, comme ses contemporains du IV ${ }^{e}$ s., avait vu se développer le culte des martyrs les souffrances du saint ont autant mis en évidence " son courage de vaincre, sa patience d'attendre, sa sérénité d'endurer ». Après avoir souligné une fois encore les vertus incomparables du saint, Sulpice réitère sa lamentation funèbre en revenant sur son chagrin et son désarroi. La suite se veut réconfortante : désormais Martin les protègera, lui et son compagnon; toutefois Sulpice a bien conscience des fautes qui l'alourdissent encore pour suivre le même chemin que le saint. Il attend la venue prochaine d'Aurèle et termine par une formule typique du genre épistolaire, à savoir que sa lettre soit une sorte de « conversation » avec son ami.

La lettre à Bassula apporte comme un couronnement à la vie terrestre de Martin et constitue une sorte de charnière avec sa vie dans l'au-delà, désormais comme saint Patron. L'entrée en matière se fait sur un ton de reproche : sa belle-mère est tellement 
avide de connaître le maximum d'informations sur Martin qu'elle prend les sténographes comme complices; des images fortes de pillages, de vol, de pièges désignent les manœuvres qui empêchent l'écrivain de parfaire des œuvres. Malgré tout, Sulpice lui narre une ultime anecdote de la vie de Martin et surtout un long développement sur ses derniers instants. Martin, raconte l'auteur, avait « la prescience de son décès » qu'il avait confiée à ses frères. Néanmoins son devoir d'évêque l'appelle dans la paroisse de Candes, où des clercs se querellent. Il part entouré de ses disciples et, en chemin, commande à des oiseaux chasseurs de poissons de fuir, car il voit en eux, dit-il, une image des démons qui dévorent leurs victimes.

La partie la plus importante de la lettre est consacrée aux derniers instants de Martin et à ses funérailles. Le saint est présenté entouré de ses disciples désolés, et compatit à leur chagrin de se voir abandonnés par leur pasteur, mais il se confie, ainsi que les siens, à la volonté divine. Ses derniers jours sont consacrés surtout à la prière, dans des conditions physiques éprouvantes, mais l'âme tendue vers le ciel. Il chasse définitivement le diable par sa foi en Dieu. A sa mort, le saint paraît déjà marqué par la gloire de la résurrection. Une foule immense accourt de toutes parts à ses funérailles, dont un grand nombre de moines. Puis c'est avec l'image biblique du pasteur menant ses troupeaux que vient l'énumération de la multitude des chrétiens venus assister à la gloire du saint : vieux et jeunes, vierges partagées entre la douleur et la joie. Tous sont unanimes à chanter des hymnes jusqu'au lieu de la sépulture ${ }^{5}$. Le développement s'achève solennellement par un parallèle entre ces funérailles et une cérémonie de triomphe. Du haut du ciel, désormais, que le saint Patron les protège, lui et sa lectrice, ou plutôt tous ceux qui liront ces lignes.

Tout au long de ces écrits, Sulpice nous a informés de ce qu'il considère comme les faits les plus saillants de la vie du saint. En réalité, il présente ses différents actes comme la réalisation de "vertus" que nous allons analyser désormais. S'il est parfois difficile de faire le départ entre les vertus ordinaires d'un chrétien et les vertus surnaturelles du saint, nous examinerons d'abord les premières avant de nous attacher aux secondes.

J. Fontaine a remarqué qu'une constance extraordinaire se manifeste dans les vertus de Martin 6 , de l'enfance à la vieillesse, selon les normes aristotéliciennes de l'ethos : ce sont des règles qu'observaient des biographes profanes comme Plutarque ou Suétone. Les vertus de Martin sont énumérées essentiellement au moment de sa jeunesse dans l'armée, puis dans la conclusion de la Vita, enfin dans la lettre à Aurèle ${ }^{7}$. Durant sa vie de militaire, «il montrait envers ses camarades une grande gentillesse, une charité extraordinaire, et surtout une patience et une modestie surhumaines. Car point n'est besoin de faire l'éloge de sa sobriété » (Vita 2.7). Dans le portrait du saint qui conclut la Vita, nous voyons le prolongement de telles qualités : «Jamais il n'y avait que le Christ sur ses lèvres, que la bonté, la paix, la miséricorde en son cœur» (27.1-2). Dans la lettre à Aurèle, on relève encore "le courage de vaincre, la patience d'attendre, la sérénité d'endurer. Ah! vraiment! Quel homme d'une bonté, d'une piété, d'une charité indicibles! » (13-14).

30 Sans prétendre faire une analyse exhaustive de ses vertus chrétiennes,on constate que son humilité se démontre dès son service militaire, quand il échange les rôles entre son esclave et lui, quand Hilaire à Poitiers ne peut le nommer que comme exorciste, quand à Tours, avant sa proclamation comme évêque, ses futurs confrères méprisent son aspect pitoyable et quand, une fois nommé, il garde «même humilité dans son cœur, même pauvreté dans son vêtement " (10.2); et même dans ses derniers instants, il n'accepte comme couche que la cendre et la cilice (Ep. 2.14). Que dire de sa sobriété, qui s'exerce dès 
sa vie de militaire (2.7), qui se concrétisera dans la règle qu'il observe avec ses frères à Marmoutier (10.7) et qui se retrouve dans sa pratique coutumière de l'abstinence et du jeûne (26.2) ? Si sa fermeté et sa faculté de discernement sont surtout flagrantes devant les assauts et les tromperies du diable, elles se manifestent tout à fait humainement devant les individus et les foules païennes, devant les hérétiques et leurs persécutions, devant le César Julien autant que devant l'usurpateur Maxime ${ }^{8}$.

Intéressons-nous plus spécialement à la vertu de charité : on peut dire qu'il en manifeste toutes les nuances, depuis l'épisode bien connu d'Amiens envers un pauvre qui a été qualifié de "charité » (Vita, 3), en passant par la gentillesse envers ses compagnons d'armes, l'affection qui le lie à des personnalités éminentes comme Hilaire à Poitiers, à des amis comme Sulpice, à ses frères et à ses ouailles, sa miséricorde envers les pécheurs qui est encore plus remarquable (Vita 26.5) ; cette charité, précise Sulpice avec amertume, "même en un siècle froid où elle se refroidit chaque jour jusque chez les saints, a néanmoins persévéré jusqu'à la fin en grandissant de jour en jour » (Ep.2.14).

Toutefois toutes ces vertus se nourrissent des liens privilégiés entre le saint et Dieu, essentiellement par le truchement de la prière qui ponctue toute la vie de Martin. C'est de cette forme chrétienne de l'ascétisme que découlent nombre de vertus qui transcendent l'ordinaire et dont les effets se manifestent par les avertissements divins sous forme de songes ou d'apparitions'. La familiarité avec les anges est un des traits qui caractérisent sa piété, mais le diable assaille aussi le saint de visions et d'apparitions trompeuses. Cette tension entre les forces du bien et les forces du mal -que les Anciens avaient traduite par le fameux apologue d'Hercule entre le Vice et la Vertu- est illustrée à la façon chrétienne par l'apparition du Christ s'identifiant au pauvre de la porte d'Amiens, au début de la Vita, et la vision de Satan déguisé en Christ-empereur, vers la fin.

Dans un second temps, examinons les pouvoirs surnaturels de Martin, ses uirtutes spéciales que le vocabulaire chrétien, depuis Tertullien, comme l'a rappelé R. Braun, emploie aussi pour désigner les miracles ${ }^{10}$. On sait qu'Arnobe avait notamment développé assez longuement les miracles du Christ et ceux de ses disciples pour prouver la divinité de Jésus aux païens (Adversus nationes I.42-57).

Chez Sulpice la narration du premier miracle de Martin est consacrée à la résurrection d'un catéchumène, à Ligugé. Ce dernier s'était joint à la communauté, attiré par les « règles de vie d'un si saint homme ». Martin, s'étant absenté momentanément, le retrouve décédé à son retour, entouré des frères. Accablé de douleur, le saint fait sortir les frères, s'étend sur le cadavre et prie. Deux heures après, le défunt revient à la vie; Martin clame alors son action de grâces; les frères rentrent pour constater la résurrection. Ainsi le catéchumène put recevoir le baptême et, ajoute Sulpice, «fut le premier chez nous à donner matière aux vertus de Martin et à en témoigner ». Et il souligne: "C'est à partir de ce moment que, pour la première fois, le renom du bienheureux prit de l'éclat: ainsi, celui que tous tenaient déjà pour saint, fut aussi tenu pour un homme puissant et vraiment digne des apôtres » (7.1-7). Ce premier miracle, un des plus détaillés, avec le décor, les acteurs, le processus de résurrection et la compassion profonde qui sous-tend la scène, s'apparente plutôt à la résurrection de Lazare dans le Nouveau Testament et en partie à celle du fils de la Sunamite par le prophète Elisée au second Livre des Rois (4.32.34). Un deuxième exemple de résurrection est donné à propos d'un petit esclave qui s'était suicidé chez un certain Lupicin, «un notable selon ce monde » (8.1-3). 

illustré. «La grâce des guérisons, écrit Sulpice, était chez lui si puissante que presque aucun malade ne l'approcha sans recouvrer aussitôt la santé » (16.1). Cinq exemples en sont donnés : le plus long développement concerne la guérison d'une jeune paralysée à Trèves selon un scénario très proche des Evangiles avec une description de la malade, de l'affliction des proches ; aux demandes réitérées du père qui a foi en Martin, pressé aussi par les évêques qui l'entourent, ce dernier après avoir longuement prié guérit la jeune fille (16.2-7). Chez les Parisiens, la guérison d'un lépreux comporte aussi de fortes connotations évangéliques (18.3-4). Puis Sulpice énumère trois autres miracles : la fille de l'ancien préfet Arborius est guérie de la fièvre par le truchement d'un objet venant de Martin (19.1-2) ; Paulin est guéri d'une ophtalmie (19.3) ; Martin lui-même est soigné par l'intermédiaire d'un ange (19.4). paganisme des campagnes. Chez les auteurs chrétiens latins, depuis Tertullien jusqu'à Saint Augustin, la lutte contre l'idolâtrie avait été très vive et les chrétiens avaient repris et développés les arguments qu'un Isaïe par exemple avait déjà utilisés dans l'Ancien Testament ${ }^{11}$. La première anecdote concernant cette opposition de Martin aux cultes païens ne sert qu'à montrer les pouvoirs du saint capable de figer les acteurs d'un enterrement et de les mouvoir à son gré $(V i t a, 12)$; l'épisode du pin abattu est plus explicite car il suit la destruction d'un temple dans un village et aboutit à la conversion des assistants (Vita 13.1-8) et Sulpice donne alors une conclusion plus générale en constatant que « les vertus et l'exemple de Martin donnèrent tant de force [au nom du Christ] qu'il ne se trouve [en ces pays] plus un seul endroit qui ne soit rempli d'églises ou d'ermitages en très grand nombre» (Vita, 13.9). Deux autres exemples soulignent les pouvoirs surnaturels du saint; ils concernent encore la destruction de temples païens : dans le premier cas, Martin freine l'action du feu qui menaçait des édifices (Vita, 14.1-2); dans le second, à Levroux, il affronte une foule de païens qui s'opposaient à la destruction d'un sanctuaire (Vita.14.3-7).Toutes ces actions vont de pair avec les attaques que les auteurs chrétiens n'avaient cessé de porter contre l'idolâtrie et que les édits impériaux allaient concrétiser de plus en plus sévèrement jusqu'à la fin $\mathrm{du} \mathrm{IV}^{\circ} \mathrm{s}$.

Mais l'un des leit-motivs qui sous-tendent souvent l'action de Martin devenu chrétien est sa lutte contre le ou les démons : depuis son premier voyage missionnaire au départ de Poitiers (Vita, 6.1-2) jusqu'à ses derniers instants au milieu de ses frères (Ep. 3.16), le saint est affronté à celui que Sulpice appelle d'abord «l'Ennemi» et, à la fin, «Brute sanglante». C'est d'ailleurs sous la forme d'un dialogue âpre au style direct que ces confrontations sont évoquées dans ces deux cas extrêmes.

A plusieurs reprises, le diable apparaît à Martin sous des formes trompeuses (à la façon dont les divinités du mal comme les Furies se présentaient parfois chez les auteurs profanes). Ainsi Sulpice évoque diverses métamorphoses du diable pour tromper Martin : il se présente par exemple « sous les traits de Jupiter, la plupart du temps en Mercure, souvent même en Vénus et en Minerve " (Vita 22.1) ${ }^{12}$. Il apparaît aussi travesti en Christempereur (Vita, 24.4-8). Mais jamais il ne parvient à tromper le saint qui « le voyait de ses yeux si facilement et si distinctement qu'il le reconnaissait sous n'importe quel aspect, qu'il conservât sa nature propre ou qu'il se transformât, prenant les diverses figures du mal spirituel» (Vita, 21.1). Parfois c'est une foule de démons qui l'accable de ses invectives. L'un des épisodes les plus remarquables de ces confrontations est le dialogue entre le saint et le diable où, aux fautes anciennes de certains moines rappelées par Satan, 
Martin oppose la miséricorde du Seigneur qui atteindrait même son ennemi si ce dernier se repentait (Vita, 22.3-5) !

Quelques scènes d'exorcisme illustrent aussi les pouvoirs de Martin (qui avait reçu d'Hilaire cette fonction ecclésiale) : outre les exemples du Nouveau Testament, ceux des auteurs latins chrétiens depuis Tertullien insistaient sur ce pouvoir spécifique des Chrétiens ${ }^{13}$. Sulpice donne l'exemple de la délivrance de trois possédés : à Trèves, c'est un jeune esclave du proconsulaire Tétradius (Vita, 17.1-4) ; chez un père de famille, c'est un cuisinier (Vita 17.5-7) ; une autre fois, Martin commande au démon qui a pris possession d'un individu de déclarer si la nouvelle d'une invasion barbare est vraie: en fait dix autres démons l'ont assisté pour répandre de faux bruits (Vita, 18.1-2) : on assiste ici en quelque sorte à la christianisation des méfaits produits par Fama ou Pauor chez les Anciens. L'un des épisodes les plus développés illustrant ce pouvoir de Martin concerne « une histoire merveilleuse » servant de mise en garde (Vita, 22.6) : elle a pour héros le frère Clair, particulièrement vertueux, dans un ermitage proche, qui est confronté à un certain Anatole ; ce dernier feint d'avoir des pouvoirs surnaturels et donne pour preuve de sa «sainteté » un merveilleux vêtement blanc dont il est revêtu; Clair demande alors aux frères de prier pour savoir la vérité; mais voici que lorsqu'il veut entraîner le faux prophète chez Martin, l'autre résiste et crie tandis que le beau vêtement disparaît (Vita, 23.1-10)!

Toutes ces vertus de Martin s'organisent particulièrement autour du thème de la militia Dei ou militia Christi : dès le Livre de Job (Vulg.7.1), cette image caractérisait la vie de l'homme sur terre. Avec l'Epître aux Ephésiens de Saint Paul (6.10-17) ce thème avait pris une couleur chrétienne et avait été abondamment exploité par les auteurs latins chrétiens de Tertullien à Prudence, dont on se rappelle la fameuse formule célébrant des martyrs militaires : Caesaris uexilla linquunt, eligunt signum crucis (Périst. I.34) ${ }^{14}$. Pour Martin ce thème est particulièrement approprié, vu son long service dans l'armée. Toutefois, pensant aux détracteurs du saint, Sulpice a souligné que dès son enfance Martin aspirait au service de Dieu (Vita 2.2), que c'est sous la contrainte qu'à l'âge de 15 ans il a été « lié par les serments militaires " (Vita 2.5), et l'auteur donne un éclat particulier à l'entrevue de Martin avec le César Julien pour demander son congé notamment quand il lui déclare : «Jusqu'ici, j’ai été à ton service : permets-moi maintenant d'être au service de Dieu (...) ; moi, je suis soldat du Christ». Plus tard, quand, une fois évêque, il tente de détruire un sanctuaire païen, «deux anges armés de lances et de boucliers se présentèrent à lui comme une milice céleste » (Vita, 14.5). Quand il guérit la paralysée de Trèves, c'est encore cette image qui est utilisée : « recourant à ses armes coutumières en semblable circonstance, il se prosterna sur le sol et pria » (Vita 16.7).

41 Dans les lettres, ce thème réapparaît avec force. Dans la première, Sulpice raconte que, menacé par le feu dans l'église d'une de ses paroisses, « Martin saisit le bouclier de la foi et de la prière »; d'abord, le diable l'avait empêché de « combattre le danger par la foi et la prière »; puis, il avait « repris l'étendard de la croix et les armes de la prière » (13-15).

Dans la deuxième lettre, à sa mort, "Martin vient de se voir remettre la couronne de justice, après avoir définitivement vaincu le monde et triomphé du siècle » (7). S'il avait vécu lors des persécutions, Martin aurait cherché «la palme du sang» (10). Sulpice évoque encore « les divers combats de chaque jour, qu'il livra contre la puissance du mal humain et du mal spirituel » (13). adressée par Martin à Dieu avant sa mort file constamment la métaphore militaire : 
"C'est un lourd combat que nous menons, Seigneur, en te servant dans ce corps; en voilà assez des batailles que j'ai livrées jusqu'à ce jour. Mais si tu m'enjoins de rester en faction devant ton camp pour continuer à accomplir la même tâche, je ne me dérobe point... Tant que tu m'en donneras l'ordre toi-même, je servirai sous tes enseignes. Et bien que le souhait d'un vieillard soit de recevoir son congé... mon courage demeure pourtant victorieux des ans » (13). La conclusion de la lettre sur un ton solennel est consacrée aux obsèques du Saint comparées à une cérémonie de triomphe (21).

Plusieurs remarques s'imposent après nos diverses analyses concernant ces deux types d'œuvres de Sulpice consacrées à Martin. Il faut aussi s'imprégner de la lecture des Dialogues rédigés vers 404, qui se déroulent entre Sulpice, Postumianus revenant de l'Orient et Gallus disciple de Martin, lors desquels il est question de l'immense succès de la Vita dans le monde et surtout, encore et toujours, des actions et des vertus remarquables de Martin ${ }^{15}$. Une partie du livre II de la Chronique de Sulpice ajoute encore des détails à la vie du Saint.

Constatons encore que la vie de Martin s'insère dans un contexte de développement inouï du christianisme soutenu, il faut le rappeler, par le pouvoir impérial : les plus grands auteurs latins chrétiens du siècle témoignent des mêmes aspirations spirituelles par leurs actes et leurs écrits. La lutte contre le paganisme? Elle est illustrée brillamment par l'intervention de Saint Ambroise lors de l'affaire de l'Autel de la Victoire à Rome. Saint Augustin s'y livrera encore au début du $\mathrm{V}^{\mathrm{e}} \mathrm{s}$. dans la Cité de Dieu. La lutte contre l'arianisme? Elle a été menée par Hilaire à Poitiers, Ambroise à Milan, Augustin à Hippone. La vocation monastique? Elle est surtout représentée par Jérôme, alors que Hilaire, Ambroise et Augustin doivent veiller à leurs fonctions épiscopales.

Mais il faut accorder aussi une attention particulière à l'expansion extraordinaire du culte du Saint après sa mort, dont on peut dire qu'il constitue un vrai « miracle », dû à la ferveur du peuple et de l'aristocratie, ferveur qui s'est poursuivie intensément durant le Moyen Age : un nombre extraordinaire de paroisses en Gaule et au-delà se sont placées sous le patronage de Martin, tandis que se multipliaient les pèlerinages à Tours.

Sur le plan littéraire, la Vita Martini restera selon J. Fontaine "pour un millénaire occidental l'archétype admiré de toute œuvre hagiographique $»^{16}$. Deux évêques ont contribué à développer -si possible en l'embellissant et en la complétant- la Vie de Saint Martin d'après les œuvres de Sulpice Sévère : ce furent les évêques de Tours Perpetuus au $\mathrm{V}^{e}$ s. et Grégoire au $\mathrm{VI}^{\mathrm{e}} \mathrm{s}$. qui suscitèrent ou favorisèrent grandement le premier la transposition en vers de la Vita Martini par Paulin de Périgueux, et le second celle réalisée par Venance Fortunat, deux œuvres dont S. Labarre a donné une belle analyse dans sa thèse sur Le manteau partagé (Paris, 1998). N'omettons pas non plus les deux beaux volumes édités aux Mémoires de la Société archéologique de Touraine (tome 62 et 63), le premier rassemblant 12 conférences martiniennes tenues à Tours d'octobre 96 à octobre 97, le second les Actes du Colloque universitaire tenu également à Tours du 22 au 25 octobre 1997 auquel participaient une trentaine de conférenciers pour célébrer le $16^{\text {ème }}$ centenaire de la mort de Saint Martin.

Il est remarquable que le thème choisi pour ce Colloque ait été celui du partage du manteau, dont J. Fontaine a donné une étude littéraire approfondie, et dont il a été remarqué que cette scène célèbre avait, dès les origines, été choisie par des milliers d'artistes qui, au fil des siècles, ont représenté le Saint faisant ce geste si symbolique de celle qui reste, en fin de compte, la première de ses vertus, comme elle l'est de toutes les autres pour tous les chrétiens : la charité. 


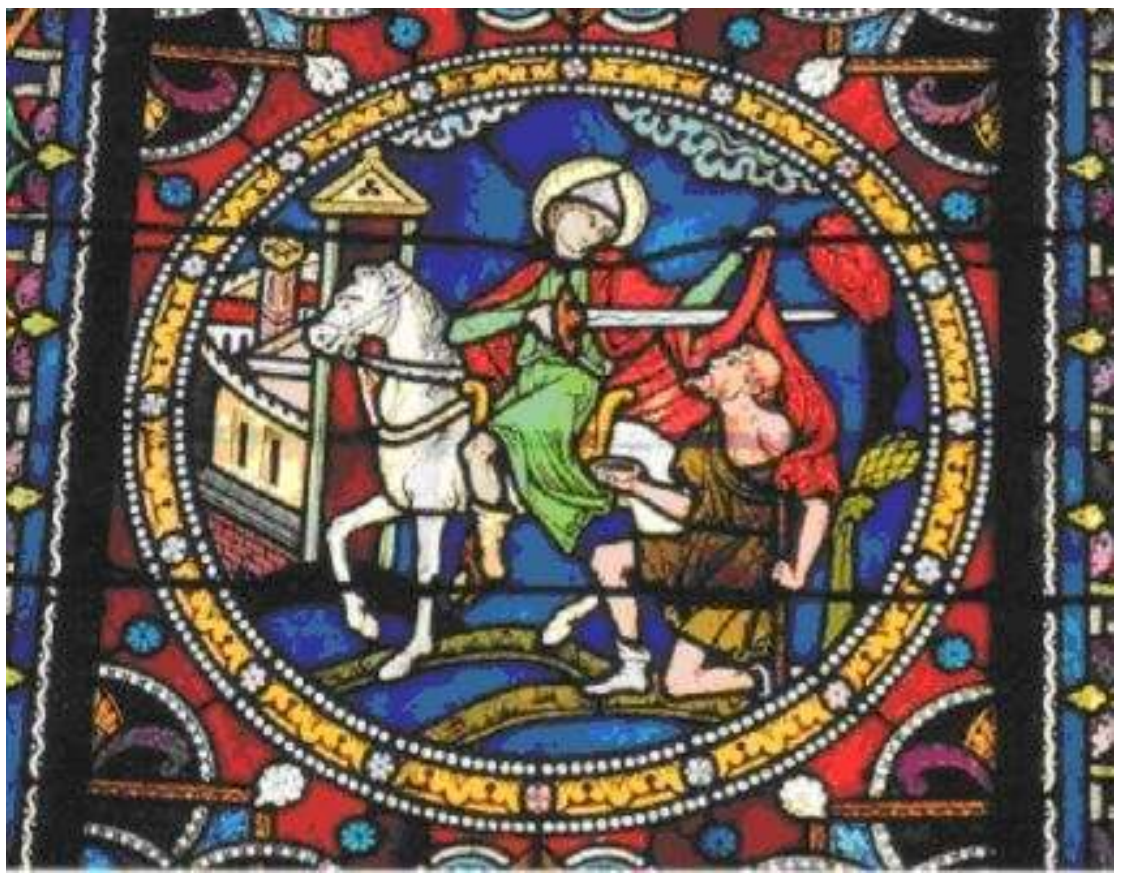

\section{BIBLIOGRAPHIE}

Amat J., 1985, Songes et visions : l'au-delà dans la littérature latine chrétienne, Paris.

Braun R., 1962, Deus Christianorum, Paris.

Brown P., 1971, La vie de Saint Augustin, Paris.

Daniélou J. et Marrou H.I., 1963, Nouvelle Histoire de l'Eglise, Paris.

Fontaine J., 1980, Etudes sur la poésie latine tardive d'Ausone à Prudence, Paris.

Fontaine J., 1967-1969, Sulpice Sévère, Vie de Saint Martin, Paris, S.C. 133,134 et 135.

Fredouille J.C. (et Zehnacker H.), 1978, Littérature latine, Paris.

Labarre S., 1998, Le manteau partagé, Paris, I.E.Aug.

Pietri L., 1975, Saint Martin, Textes de Sulpice Sévère, Suplt Lettre Ligugé (jt-oct.).

Testard M., 1978, Chrétiens latins des premiers siècles, Paris.

Von Harnack, 1905 (réimpr.1963), Militia Christi : sur le Christianisme et le service militaire des 3 premiers siècles, Tübingen, Darmstadt.

1997, XVI centenaire de la mort de Saint Martin, Mémoires de la Société archéologique de Touraine, Tomes LXII et LXIII, Tours. 


\section{NOTES}

1. Daniélou et Marrou 1963 ; Fredouille 1978 ; Testard 1978.

2. D'ailleurs son ami Paulin de Nole écrit parallèlement nombre de poèmes célébrant Saint Félix en évoquant sa vie et ses miracles (395-408).

3. Il a alors une quarantaine d'années si l'on songe que le service dans l'armée durait 25 ans.

4. Il sera exilé en 356 avant de revenir dans son diocèse en 360 jusqu'à sa mort en 367 . Avec Ambroise, Jérôme et Augustin, c'est un des grands noms de la théologie et de la littérature chrétiennes de l'époque.

5. Ceci correspond à l'essor de l'hymnique latine chrétienne sous l'influence d'Hilaire et d'Ambroise

6. Cette constance est signalée expressément dans la Vita (10.1): idem enim constantissime perseuerabat qui prius fuerat.

7. On peut aisément y retrouver les vertus théologales et cardinales!

8. Cf $4.5 ; 5.5 ; 6.4 ; 13.4 .8 ; 20.1$.

9. J.Amat (1985) en a analysé les principaux exemples.

10. Deus Christianorum, Paris, 1962, p. 108.

11. En plein $\mathrm{IV}^{\mathrm{e}} \mathrm{s}$., on se rappelle que l'affaire de l'Autel de la Victoire à Rome avait symbolisé clairement cette lutte.

12. Ce qui rejoint la polémique des Apologètes qui soulignaient l'identité entre les dieux païens et les démons.

13. Tertullien développe ce thème (Apol. 23.5), et, à l'époque de Martin, Hilaire, puis Jérôme décrivent ces phénomènes (cf. Amat 1985 : 352).

14. Cf. Von Harnack 1905.

15. Le Dialogue représente une forme littéraire différente de l'hagiographie et de la lettre et rejoint donc le souci de l'auteur de ne pas lasser le lecteur. Par ailleurs, Sulpice y constate qu'on évince des auditeurs qui seraient poussés plus par la curiositas que par la pietas. On retrouve des constantes telles que la ferveur et la fermeté de Martin, son pouvoir sur les démons et la nature, la pratique de l'ascèse et de la prière. La nouveauté consiste en quelques indications sur les contacts avec des femmes pieuses.

16. Etudes..., 1980, p. 29.

\section{RÉSUMÉS}

En 397, Sulpice Sévère fait paraître une Vita Martini qui connut un grand succès, et construit l'image de Martin Saint d'Occident, rivalisant avec l'image d'Antoine, telle qu'Athanase l'avait conçu comme Saint d'Orient, par la Vita qu'il avait composée à son sujet. La Vita Martini est d'abord un discours d'éloge où il s'agit principalement de uirtutes aeternare; de 397 à 398 trois épîtres adressées à des admirateurs de Martin complètent ce travail parénétique entrepris par Sulpice Sévère. Dans ces deux genres d'écriture, le souci de la vérité prévaut sur celui du beau style et s'affirme par la christianisation de l'idéal profane des exempla. L'auteur fait l'apologie des victoires remportées par Martin triomphant de l'arianisme, du paganisme, du diable et des démons. Les trois épîtres rédigées après la mort de Martin insistent particulièrement sur l'action 
du Saint thaumaturge et sur sa mort qui s'intègre au culte des martyrs déjà répandu en Occident dans lequel Sulpice Sévère espère trouver son propre apaisement spirituel. Grâce à ces textes, l' éthos chrétien acquiert une nouvelle splendeur où les vertus ordinaires de l'homme chrétien diffèrent peu des vertus surnaturelles du Saint. Parmi elles, la charité qui se nourrit de la prière dans la double pratique de la militia Dei et de la militia Christi. Sulpice Sévère contribue donc au développement du christianisme soutenu par le pouvoir impérial ; il permet la diffusion du culte de Martin dont hériteront au VI ${ }^{\mathrm{e}}$ siècle. Grégoire de Tours et Venance Fortunat, auteur lui-même d'une Vita Martini versifiée qui consacrera le paradigme du «manteau partagé » pour illustrer l'essentiel de la vie de Martin.

In 397, Sulpice Severe successfully published a Vita Martini. He builds the image of Martin, Saint of the Occident and thus rivals with the image of Antony, Saint of the Orient in the Vita written by Athanase. The Vita Martini is essentially a discourse of praise whose aim is uirtutes aeternare. This work is completed with three letters written from 397 to 398 to admirers of Martin. The search for truth prevails over the beauty of style : the profane ideal of exempla is christianized. The author praises the victories of Martin against arianism, paganism, evil and demons. The three letters written after the death of Martin insist on the actions of the Saint and his death. In these texts, the Christian "ethos" takes a new dimension where the ordinary virtues of the Christian man are not very different from the surnatural virtues of the Saint. The most important of these virtues is charity. Sulpice Severe contributes to the development of Christianism and of the cult of Martin.

\section{INDEX}

Mots-clés : empire romain d'Occident, hagiographie, Saint Martin, Sulpice Sévère, Venance Fortunat, vertus chrétiennes 